\title{
DESIGN OF THE MANIPULATOR CONTROL UNIT FOR MODERNITY MARS ROVER
}

\author{
P. Ptak ${ }^{*}$, K. Sokól ${ }^{* *}$, D. Cekus ${ }^{* * *}$
}

\begin{abstract}
In this paper the design process of the manipulator control unit for Modernity II rover is presented. The rover was used in University Rover Challenge 2018 organized by Mars Society in Utah, USA and finished the challenge in the first place. The design of the present version of the manipulator (V5) required initial project, prototype design, experimental tests, mechanical and electronical improvements. The paper covers the requirements of the manipulator design and the procedures of selection of the elements for the control unit.
\end{abstract}

Keywords: Mars rover, manipulator, control unit, PCB

\section{Introduction}

The manipulator was designed on the basis of the general regulations contained in the book of rules of the University Rover Challenge (2018) competition. The main task of the manipulator is to: lift different objects; switch different types of buttons, sliders, switches; keyboard support; pouring liquid from the canister to the tank. It is expected that the manipulator will have as greatest mobility and capacity as possible, with the lowest possible weight. In the Fig. 1. the Modernity II rover with the manipulator of the PCz Rover Team (2019) is presented. The design of the described manipulator was developed for 5 years. The presented V5 version took 500 man-hours to finish.

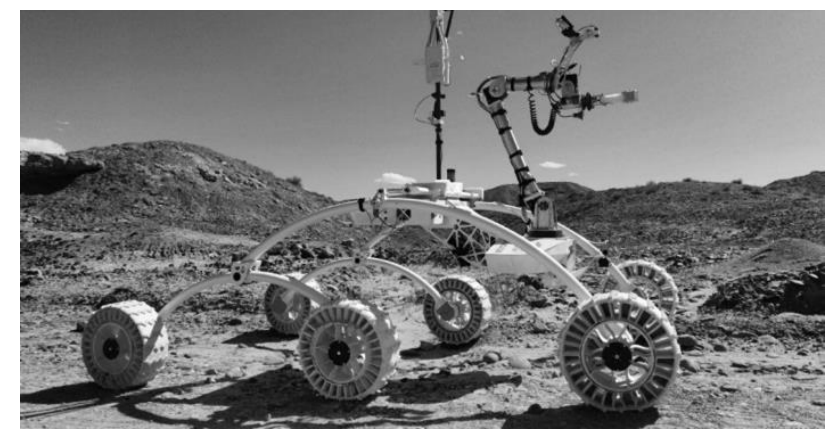

Fig. 1: Modernity II rover at URC 2018

The manipulator prepared for the University Rover Challenge is facing limitations in the form of weight and costs. It can not be compared with industrial manipulators that do not have such limitations and additionally their tasks are strictly defined. The competition regulations do not specify in detail what will be done with the help of the manipulator except the maximum lifting weight $(5 \mathrm{~kg})$. It is a kind of a

Piotr Ptak, MSc., Institute of Mechanics and Machine Design Foundation, Częstochowa University of Technology Dąbrowskiego 73, 42-200 Częstochowa, Poland, ptak@imipkm.pcz.pl

** Krzysztof Sokół, PhD.: Institute of Mechanics and Machine Design Foundation, Częstochowa University of Technology Dąbrowskiego 73, 42-200 Częstochowa, Poland, sokol@imipkm.pcz.pl

*** Dawid Cekus, Assoc Prof.: Institute of Mechanics and Machine Design Foundation, Częstochowa University of Technology Dąbrowskiego 73, 42-200 Częstochowa, Poland, cekus@imipkm.pcz.pl 
challenge to build the manipulator as light as possible and as universal as possible for any encountered situation that can happen on University Rover Challenge or on Mars.

\section{Design of the manipulator}

\subsection{Mechanical design}

The manipulator in mechanical terms is a structure made of aluminum profiles that ensure the appropriate ratio of mechanical strength to mass. It is a 3 -segment motion system with the possibility of clamp and rotation of the last part. The manipulator has 5 degrees of freedom. Actuators on the manipulator have been used as drive units. They are brush motors with built-in planetary gears, increasing torque and decreasing the output velocity. On the basis of calculations of torques and velocity, drive sets were individually selected for each degree of freedom. The first 4 degrees of freedom from the base have attached to the outputs of planetary gears the worm gears, which additionally provide self-locking of all 4 degrees of freedom. The end of the 3rd member has a clamp, which also has a rotation function. Both of these motions are realized without additional gears. As presented by Pierzgalski, Ptak, Cekus and Sokó (2017) the similar studies on rover's elements have been done in the past taking into account the durability of the manipulator and frame.

The major mechanical corrections concerned the minimization of clearances between the manipulator members. It was the biggest test problem, amplifying all electronic-programming inaccuracies. The accuracy of the potentiometers serving as information sources for angular positions of all the elements was electronically increased, the ADC's microprocessor resolution was increased through an indirect ADC converter, which improved the reading accuracy by 64 times. Disturbances resulting from the operation of brush motors and their controllers influenced the accuracy of voltage readings at the processor's ADC inputs. The signal lines were protected against such interference using shielded cables and implemented RC filters selected experimentally, which significantly reduced the noise observed on the ADC voltage reading. During tests, a significant improvement in the accuracy of the manipulator's action was observed, what can be seen in the movie (2018) where one can see how to write a text message. Each of the corrections made had a positive effect on the precision of movements.

\subsection{Electrical and programming design}

The power source for electronics on the rover are $6 \mathrm{~S}$ lithium polymer batteries. These are the batteries that give a nominal voltage of $22.2 \mathrm{~V}$, with a total capacity of $10000 \mathrm{mAh}$, which allows the entire vehicle to work for more than 2 hours. Batteries of this type were used because of the best capacity to weight ratio.

To control all drives, the single-channel H-bridges were used that operate in the voltage range 5.5-30V. They are able to give up to $20 \mathrm{~A}$ of continuous current. They have a TTL communication interface a serial connection with a microcontroller. Their maximum PWM signal frequency is $21 \mathrm{kHz}$. Not all drives consume currents of 10-20A, it was possible to use less efficient current brush motor controllers. However, it was decided due to the unification of components used for the project, service issues, and the ability to easily adapt the controller to other applications.

In order to optimize movements and shorten the operation time, inverse kinematics was used. Motion equations were implemented based on the lengths of the manipulator's members and the angular relationship between them. Thanks to this, the operator does not have to think about the movement of individual elements to perform operations, and it is enough to only observe the end point of the manipulator and move it intuitively, just like with his own hand. The reverse kinematics mechanism will calculate how to set the angles between the elements to reach the expected point. The lengths of the members are known and their values are simply entered into the system of equations whereas the values of the angles between the elements should be given in real time. This was done electronically by mounting in the axis of each degree of freedom, a rotary linear potentiometer. For this application, 
potentiometers with plastic path material have been selected and the most important parameter in this case, linearity tolerance of $+/-1 \%$, which affects the accuracy of the manipulator motion.

The microcontroller reads the analog voltage value, which after initial calibration is converted into an angular value. In order to increase the reading resolution of the voltage value (maximum microcontroller reading resolution - 10 bits), ADC converters between the microcontroller and potentiometers were inserted, the resolution of which is 16 bits and the sampling rate up to 860 samples per second. The readout value of the analog voltage through the transmitter is transferred to the microcontroller using the I2C interface. This allowed not only to improve the readability, but also to better protect the measurement against interferences.

In order to protect the manipulator against collision between its members, the values of angles from the potentiometers were used, calibrating their possible extreme values. In order to increase the reliability of this function, magnetic switches have been used which also protect against the collisions.

Such a protections as the extreme potentiometer positions or limit switches of course increase security, but their failures can sometimes make it impossible to complete the task. For this purpose, it was possible to disable any limit switch or potentiometer remotely from the application on the computer. This mode of operation does not protect the manipulator from the collision, but the operator has a chance to do the job as desired.

During the manipulator operation, most tasks are performed when the last member (gripper) is oriented vertically or horizontally, intermediate states are rarely used. Due to the fact that a fault in any potentiometer would cause the reverse kinematics to not be able to position the gripper at the desired angle, protection against such a situation was used in the form of a three-axis accelerometer installed permanently to the gripper. An analog accelerometer was selected for the project which sends 3 voltage values for 3 axes - X, Y, Z. An analog voltage values as well as voltage values from potentiometers are read by an external analog-digital converter with a resolution of 16 bits and transmitted to the microcontroller of manipulator control unit. The accelerometer requires the initial calibration as well as the potentiometer, but there is no need for corrections at a later time.

The electronic implementation of the problem of damage to the object being lifted was based on the measurement of the current consumed by each of the manipulator drives. Between all drives and their controllers, a current measurement array was added, which is based on the Hall effect. On the basis of the size of the current flowing through the chip, a proportional voltage is sent to the controller. Inside the microcontroller there is a proper interpretation of the analog voltage, which has been experimentally converted into the clamping force or the weight of the object being lifted. The function, apart from determining the force value, allows the operator to set maximum values of the current (force) that can be given to the selected motor. This prevents damaging the object being lifted, damaging any of the motors, avoiding a serious breakdowns of the manipulator. Destruction of the lifted item is unacceptable during the competition, this protection also avoids the breakdown of the vehicle in the field and the need to servicing it during a task what is punished.

The architecture of the whole vehicle control system is based on the STM32 microcontroller (MASTER), which is the manager of several ATMEGA 2560 controllers (SLAVES). Communication between controllers is based on the RS485 interface. The microcontrollers use the UART communication interface, which via chips like SP485 converts serial transmissions to differential transmission, which allows data transmission over long distances and is more resistant to interference. The vehicle's communication with the operator is carried out by the master, who uses Wi-Fi transmitters for this purpose. The operator operates the rover using a computer and controllers, performing tasks based on real-time observation of the area around the vehicle with the help of several cameras installed on it. The image from the camera is transmitted in parallel with the control signal and feedback from the vehicle.

Schematic diagram and PCB layout for the control system was designed using the Eagle CadSoft program. It is a program which supports the design of electronic circuits. It allows to create, edit diagrams of printed circuit boards. The program is very intuitive. During creation of schemas, one can use mainly 
the element library, but if needed additional libraries can be downloaded or even create by the user.

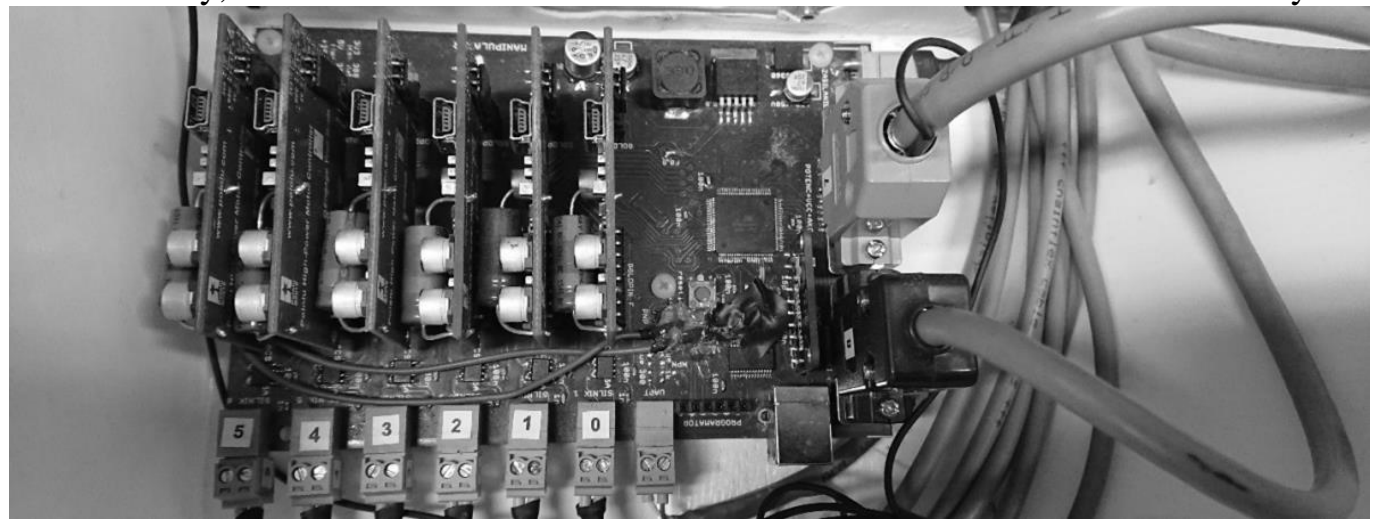

Fig. 2: Manipulator's $P C B$

In the Fig. 2 the manipulator's PCB layout is presented while in Fig. 3 the manipulator of the Modernity II rover is given.

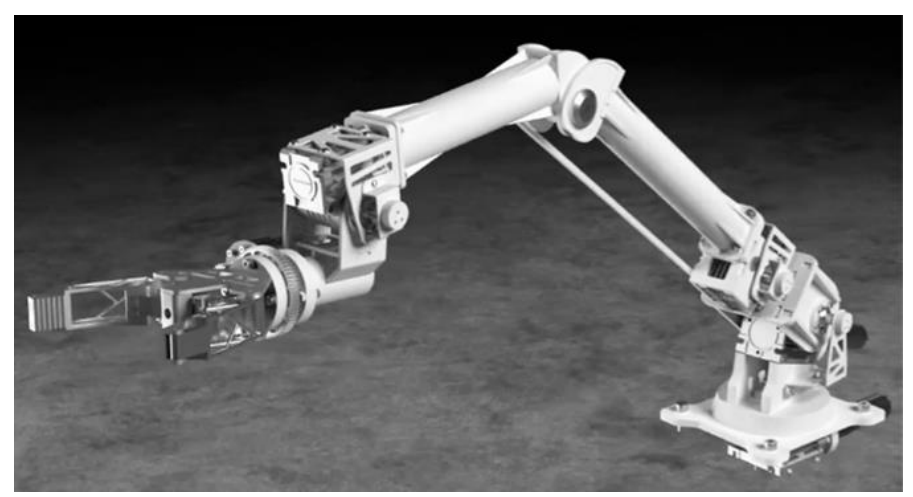

Fig. 3: Manipulator of the Modernity II rover

\section{Conclusion}

Manipulator, besides a large lifting capacity of $10 \mathrm{~kg}$ (weight of the manipulator $-11 \mathrm{~kg}$ ), protection against self-collision, failure of measuring instruments, sufficient precision achieved by large gears on planetary and worm gears, and intuitive and fast operation thanks to reverse kinematics can also handle the raised objects very gently. This feature allows operator to lift both $5 \mathrm{~kg}$ stone and an egg without damaging it.

Before the competition the manipulator was field tested. During the competition, it dealt with every task quickly and efficiently and met the constructors' expectations in 100\%, thanks to which the PCz Rover Team could win the University Rover Challenge 2018.

Currently, work is underway on the V6 manipulator design, which adopts image analysis, which is the source of information for the program implementing in some part of the tasks the autonomous work of the manipulator and in the future its fully autonomous work.

\section{References}

PCz Rover Team (2019) http://rover.pcz.pl/

Pierzgalski, M., Ptak, P., Cekus, D., and Sokół, K. (2017) Modeling and Stress Analysis of a Manipulator Mounted on a Mars Rover. Procedia Engineering, 177, pp 121-126.

Ptak, P., Pierzgalski, M., Cekus, D., and Sokól, K. (2017) Modeling and Stress Analysis of a Frame with a Suspension of a Mars Rover. Procedia Engineering, 177, pp 175-181.

University Rover Challenge (2018) http://urc.marssociety.org/home/about-urc/urc2018-scores

Manipulator presentation (2018) https://www.youtube.com/watch? $\mathrm{v}=8 \mathrm{HNwUhhus3A \& t=3 \textrm {s }}$ 